\title{
Automatic Segmentation of the Cortical Grey and White Matter in MRI using a Region-Growing Approach based on Anatomical Knowledge
}

\author{
Christian Wasserthal ${ }^{1}$, Karin Engel ${ }^{1}$, Karsten Rink ${ }^{1}$, André Brechmann ${ }^{2}$ \\ ${ }^{1}$ Otto-von-Guericke University, Magdeburg, Germany \\ ${ }^{2}$ Leibniz Institute for Neurobiology, Magdeburg, Germany \\ karsten@isg.cs.uni-magdeburg.de
}

\begin{abstract}
We propose an automatic procedure for the correct segmentation of grey and white matter in MR data sets of the human brain. Our method exploits general anatomical knowledge for the initial segmentation and for the subsequent refinement of the estimation of the cortical grey matter. Our results are comparable to manual segmentations.
\end{abstract}

\section{Introduction}

The analysis of the functional organisation of the cortical areas, as assessed by anatomical and functional MRI, requires a precise segmentation of the grey and white matter in the anatomical data. The automatic segmentation of the cortex is a complex task for two main reasons. First, the inter-subject variability of the human brain anatomy restricts the use of general anatomical knowledge. Second, image artifacts, such as partial volume effects and inhomogeneities of the scans, complicate the separation between grey and white matter regions. Several methods have been applied in recent years to estimate gray and white matter volumes (semi)automatically on MRI [1]. The most popular methods separate intensity histograms which are assumed to be composed of a grey matter distribution and a white matter distribution [2]. The data is then classified directly, or the parameters of the two distributions determine the intensity range for region growing approaches [3]. Other methods include active contours and surfaces $[4,6,5]$.

Our algorithm consists of a number of basic methods which resolve the complex task utilising general anatomical knowledge. The algorithm allows for a fully automatic and fast segmentation of the grey and white matter regions.

\section{Method}

We use an algorithm that allows for a segmentation of the grey and white matter in Talairach transformed MR data sets of the human brain.

The 3d-anatomical T1-weighted MRI were scanned on a 1.5 Tesla head scanner (GE Medical Systems). Using the commercial software BrainVoyager [7] the 
16 bit data sets have been converted to 8 bit and transformed to match the standard brain of the Talairach atlas [8] (Fig. 1(a) and 1(e)).

The result of our algorithm is a segmentation in $3 \mathrm{D}$ where each voxel $x$ of the data set is assigned one of four labels. These labels are $l_{W}$ for white matter, $l_{G}$ for grey matter, $l_{B}$ for background and $l_{V}$ for ventricles.

A histogram analysis gives an estimate for the mean value of the white matter intensity $E_{W}$, a lower intensity limit for the white matter $t_{W}$ and an upper threshold for the cerebrospinal fluid (CSF) $t_{C S F}$.

In order to get a histogram that is suitable for computing the parameters for the regions of interest, we only use voxels within an ellipsoid $\Omega_{1}$ (Fig. 1(b)), whose parameters are derived from the Talairach proportional grid (Fig. 1(a)) to give an approximation of the cerebrum.

Then, all voxels $x$ with $x<t_{C S F}$ are set to $l_{B}$. The CSF in the ventricles gets another label $l_{V}$, because the meaning of the background label does not apply here. Given the Talairach proportional grid, the ventricles can easily be located and labeled using a region-growing (Fig. 1(f)). We use two ellipsoids $\Omega_{2}$ to approximate the eye regions (Fig. 1(e)). The brightest $15 \%$ of these areas are set $l_{B}$, i.e. background, to avoid problems in the following step of our algorithm.

From our approximation of the cerebrum, $\Omega_{1}$, in combination with the calculated mean $E_{W}$, a small area within the white matter is automatically assigned as seed voxels for a second region growing process. White matter voxels are now segmented based on intensity and minimum distance to background (Fig. 1(c)). More specifically, $x=l_{W}$ if $x>t_{W} \wedge \min \left\{d\left(x, y=l_{B}\right)\right\} \geq 3 \mathrm{~mm}$. The second criterion is based on anatomical knowledge about the brain, that grey matter has a thickness of $3-5 \mathrm{~mm}$, even if the data suggest otherwise. This criterion

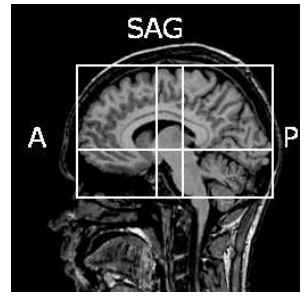

(a)

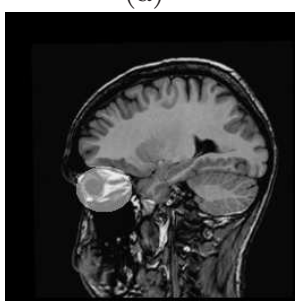

(e)

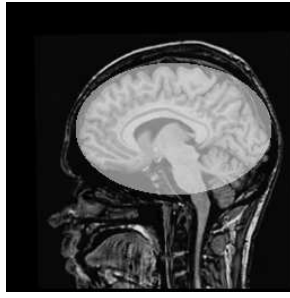

(b)

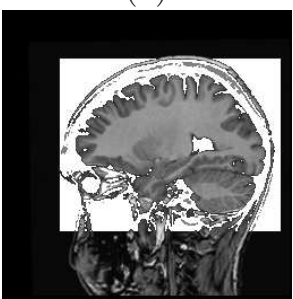

(f)

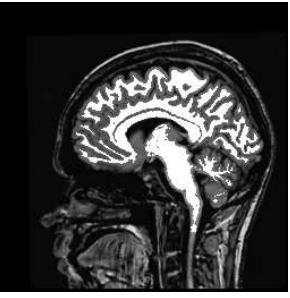

(c)

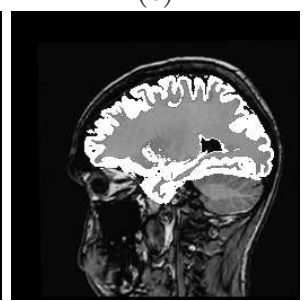

(g)

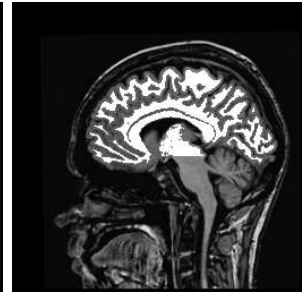

(d)

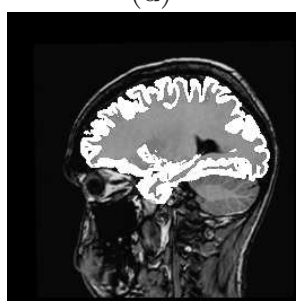

(h)

Fig. 1. Figures 1(a) and 1(e) present two sagittal slices from one of the data sets. For a detailed description of the intermediate segmentation results (Section 2) 
does not apply to the region around the ventricles, where white matter directly borders CSF. Since voxels of this region are assigned the label $l_{V}$ instead of $l_{B}$, this poses no problem to our algorithm. The masking of the eyes was necessary because these high intensity regions touch the brain.

Now we apply a flooding algorithm initialised on the bottom-most voxel $x=l_{W}$ of the data set, which is in our case always situated in the spinal cord. The algorithm counts in every axial slice $i$ voxels $x_{i}=l_{W}$ connected to the spinal cord and sets every voxel $x_{i}=l_{B}$ if $\frac{\# x_{i+1}}{\# x_{i}}<\theta$. Once the flooding reaches the cerebrum, the number of connected white matter voxels in a slice increases significantly and the algorithm stops. Because of this increase, any threshold $100<\theta<10000$ gives the same result. Note, that this still allows for white matter voxels below the final flood level in other parts of the brain. Using this simple rule we remove all voxels within the spinal cord and the cerebellum and retain a clean segmentation of the white matter (compare Figs. 1(c) and 1(d)).

In the next step, any unlabelled voxels $x$ with $\min \left\{d\left(x, y=l_{W}\right)\right\}<6 m m$ are set $l_{G}$, i.e. grey matter (Fig. $\left.1(\mathrm{~g})\right)$. This is a reasonable choice as these voxels have not been labelled as background and are still within the expected range for grey matter. All other still unlabelled voxels are set to $l_{B}$. In a number of postprocessing steps we now reduce the number of misclassified voxels. Via a connected component analysis voxels that represent fat, ventricles or meninges are identified and set to $l_{B}$. By now, we can easily calculate a mean value $E_{G}$ from a more reliable set of voxels $x=l_{G}$ and can change the label of any $x=l_{W}$ with $\left|x-E_{G}\right|<\left|x-E_{W}\right|$ to $l_{G}$ (Fig. $1(\mathrm{~h})$ ). Finally, all $x=l_{V}$ can be set $l_{B}$, so that all voxels that are not grey or white matter are now labelled as background.

\section{Results}

Five data sets were available for the evaluation. The quality of the data varied with respect to signal-to-noise ratio and grey level inhomogeneities. A gold standard was given in terms of manual segmentations of four transversal example slices per data set. Additional reference segmentations were computed by using the software BrainVoyager [7], which relies on a comparable segmentation approach and is of widespread use in the neuroimaging community. These segmentations were generated independently by one neurobiologist and one trained expert.

\subsection{Performance and Robustness in Parametrisation}

The segmentation using BrainVoyager includes the isolation of the brain by applying standard Talairach masks, as well as a histogram-based grey and white matter classification. The masking is followed by nonlinear smoothing and region growing within the segmented volume. The cortex boundary is then improved using morphological operations [3]. For reasons of comparability to our algorithm, we didn't include the inhomogeneity correction usually performed in BrainVoyager by an expert. 
For the segmentation with BrainVoyager user interactions were required in one of the five cases. If parameter values, e.g. the threshold for separating grey and white matter, were poorly specified the segmentation failed. Small variations in the parameter values led to significant alterations in the segmentations. The whole segmentation process (without any topological corrections) took the trained user about 300 seconds on a standard PC (3.2 GHz Pentium 4).

Our algorithm is fully automatic and requires no user interaction. In our approach, parametrisation of the different region growing steps is more robust, because it is less data driven but utilises anatomical information, e.g. distanceto-surface priority. In contrast to BrainVoyager, no smoothing for reducing the variation in the grey and white matter signal intensity is needed. Computational time of the whole algorithm on a data set with $256^{3}$ voxels is about 65 seconds. Note, that the postprocessing steps take about 50 seconds, while the large part of the segmentation can be computed very fast due to the simple algorithms.

\subsection{Assessment of the Segmentation Results}

A visual inspection of the segmentation results by another two experts indicate that our segmentations are of high quality even in regions of low contrast, e.g. the occipital lobe (Fig. 2).

To evaluate the accuracy of our segmentation, we assembled a quantitative analysis based on the slices of the five data sets for which a gold standard was available. Our analysis showed, that the accuracy of our segmentation is comparable with the accuracy of the manual segmentations (Table 1). In contrast, the

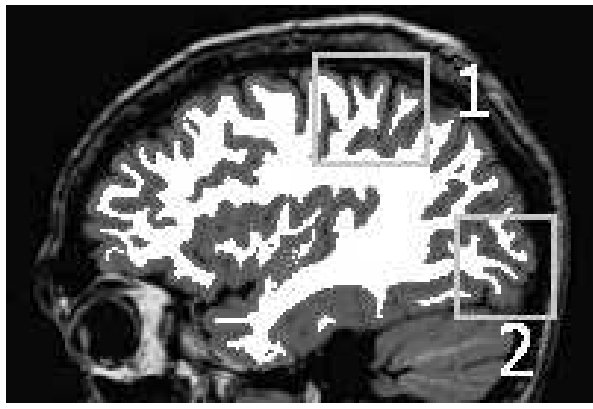

(a) Region Growing

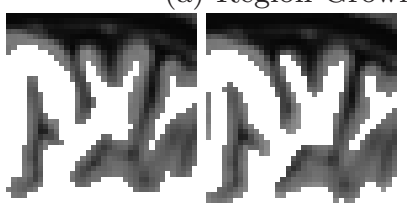

(c) RG:1

(d) manual:1

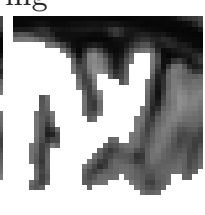

(e) $\mathrm{BV}: 1$

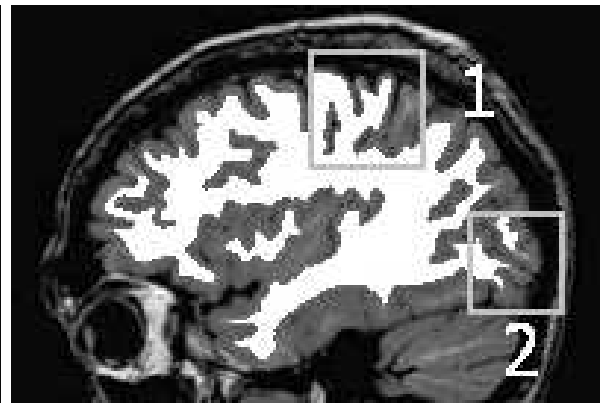

(b) BrainVoyager

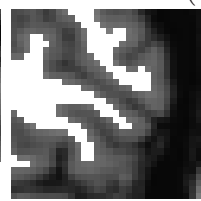

(f) RG:2

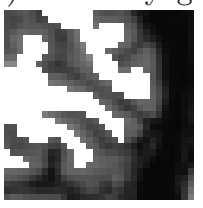

(g) manual:2

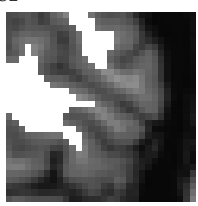

(h) BV:2

Fig. 2. Comparison of our algorithm with a BrainVoyager segmentation: representative slice $(a, b)$; particulary difficult regions from the slice $(c-h)$; further examples at http://isgwww.cs.uni-magdeburg.de/bv/rgapplett/ 
Table 1. Quantitative assessment of the segmentations using BrainVoyager and our Region-Growing approach based on anatomical knowledge. We computed the (mean \pm standard deviation of the) Hausdorff-distance $d_{H}$ and the Mean Squared Distance $d_{M}$ of the grey-white matter boundaries to compare the segmentation results with the expert's segmentations of example slices of five data sets (in $\mathrm{mm}$ )

\begin{tabular}{llccccc}
\hline & Data set & 1 & 2 & 3 & 4 & 5 \\
\hline$d_{H}$ & BrainVoyager & $17.9 \pm 7.9$ & $17.3 \pm 6.2$ & $14.0 \pm 5.7$ & $16.7 \pm 4.5$ & $17.3 \pm 4.8$ \\
& Region-Growing & $7.4 \pm 2.7$ & $7.5 \pm 2.6$ & $9.0 \pm 5.4$ & $9.3 \pm 2.0$ & $10.2 \pm 3.3$ \\
$d_{M}$ & BrainVoyager & $16.8 \pm 12.2$ & $14.5 \pm 10.7$ & $10.9 \pm 6.8$ & $21.0 \pm 6.8$ & $15.6 \pm 10.2$ \\
& Region-Growing & $1.7 \pm 0.8$ & $1.2 \pm 0.4$ & $1.5 \pm 0.5$ & $3.3 \pm 1.7$ & $3.8 \pm 0.9$ \\
\hline
\end{tabular}

BrainVoyager segmentations clearly underestimate the white matter and miss white matter of gyri which can be easily identified (compare for example Figs. $2(\mathrm{c})$ and $2(\mathrm{~d}))$.

\section{Discussion}

We presented a technique for the segmentation of grey and white matter in MRI data using a modified region growing process. By incorporating general anatomical knowledge in a sequence of simple segmentation steps we obtained robust and fully automatic segmentation results. These results are comparable to the manual segmentations of trained experts.

\section{References}

1. Pham D.L., Xu C. and Prince J.L.: Current methods in medical image segmentation. Ann Rev of Biomed Engineering 2(1) 2000:315-337.

2. Mangin JF, Coulon O, Frouin V. robust brain segmentation using histogram scale-space analysis and mathematical morphology. Lect Note Comp Sci. 1998;3175:1230-41.

3. Kriegeskorte N, Goebel R. An efficient algorithm for topologically correct segmentation of the cortical sheet in anatomical MR volumes. NeuroImage. 2001;14:32946.

4. Davatzikos C, Bryan N. Using a deformable surface model to obtain a shape representation of the cortex. IEEE Trans Med Imag. 1996;15(6):785-95.

5. Goldenberg R, Kimmel R, Rivlin E, et al. Cortex segmentation: A fast variational geometric approach. IEEE Trans Med Imag 2002;21(12):1544-51.

6. Dale A, Fischl B, Sereno M. Cortical surface-based analysis: 1. Segmentation and surface reconstruction. NeuroImage 1999;9:179-94.

7. Goebel R. Brain Voyager 2.0: From 2D to 3D fMRI analysis and visualization. NeuroImage 1997;5:635, http://brainvoyager.com

8. Talairach J, Tournoux P. Co-Planar Stereotaxic Atlas of the Human Brain. Stuttgart: Thieme 1988 